\title{
Efficacy of 5\% Tea Tree Oil Hydrogel on Healing Morbus Hansen's Chronic Plantar Ulcer
}

\author{
Marissa Astari Rubianti ${ }^{1}$, Evy Ervianti ${ }^{1}$, Muhammad Yulianto Listiawan ${ }^{1}$, Diah Mira \\ Indramaya $^{1}$, Rahmadewi ${ }^{1}$, Esti Hendradi ${ }^{2}$, Cita Rosita Sigit Prakoeswa ${ }^{1}$ \\ ${ }^{I}$ Department of Dermatology and Venereology, Faculty of Medicine, Universitas Airlangga, Dr. \\ Soetomo General Academic Teaching Hospital, Surabaya, Indonesia \\ ${ }^{2}$ Departement of Pharmaesthetics, Faculty of Pharmacy, Universitas Airlangga, Surabaya
}

\begin{abstract}
Background: The damage of the peripheral nerves that occurs in Morbus Hansen (MH) patients can cause disability due to loss of sensory, motor and autonomic functions can cause wounds, infections, ulcers and disability. Ulcers are the most common cause of disability complications in $\mathrm{MH}$ patients and requires a certain time of healing. As many as $10-20 \%$ of $\mathrm{MH}$ patients experience ulcers on the soles. Tea Tree Oil (TTO) is extracted from steam distillation of the leaves and twigs of the Australian native shrub Melaleuca Alternifolia. In ulcers, TTO can work as an antimicrobial, anti-inflammatory, and antioxidant. TTO preparation in the form of hydrogel is believed to help the wound healing process. Purpose: To investigate the effect of TTO hydrogel $5 \%$ on the healing of Chronic Plantar Ulcer of Leprosy (CPUL). Methods: Tea tree oil hydrogel $5 \%$ was applied every 3 days for up to 8 weeks in 22 subjects with chronic MH plantar ulcers. Ulcer size, side effects, and possible side effects are evaluated weekly. Result: There were significant clinical and statistical differences in ulcer size $(\mathrm{p}=$ $0.000)$ and in ulcer depth $(\mathrm{p}=0.000)$ after TTO hydrogel $5 \%$. No side effects occurred in this study. The ulcer healed was $59 \%$, the ulcer improved by $36.4 \%$, the persistent ulcer was $4.6 \%$, and there was no ulcer that got worse. Conclusion: TTO hydrogel $5 \%$ is effective in the healing process of chronic $\mathrm{MH}$ plantar pedis ulcers.
\end{abstract}

Keywords: Tea tree oil hydrogel, chronic plantar ulcers in leprosy (CPUL), morbus hansen.

Correspondence: Cita Rosita Sigit Prakoeswa, Department of Dermatology and Venereology Faculty of Medicine, Universitas Airlangga / Dr. Soetomo General Academic Hospital, Surabaya, Jl. Mayjen Prof. Dr. Moestopo No. 6-8 Surabaya 60131, Indonesia. Phone: (031) 5501609, e-mail: drcita.rosita@gmail.com

\section{BACKGROUND}

Morbus Hansen $(\mathrm{MH})$ is a disease caused by Mycobacterium leprae, primarily attacks the skin, peripheral nerves, upper respiratory mucosa, eyes and is still found to be endemic in several countries including Indonesia. Damage to the peripheral nerves that occurs in $\mathrm{MH}$ patients can cause disability due to loss of sensory, motor, and autonomic functions. The nerve damage can cause further damage due to trauma, abnormal pressure, and secondary infection resulting in tissue damage and loss.

Ulcers are the most common cause of disability complications in $\mathrm{MH}$ patients and need certain time to heal. As many as $10-20 \%$ of $\mathrm{MH}$ patients have plantar ulcers because most of the weight of the body rests on the front and the parts with bony protrusions from the soles of the feet. Untreated conditions, delayed diagnosis, and inadequate treatment lead more severe nerve damage resulting in neuropathic ulcers. Chronic ulcer is a serious medical and social problem for the patient. Patients must receive long-term care with a variety of local drugs and various dressings so that it requires a lot of money and time. Patient quality of life also will decrease. ${ }^{1}$
Standard therapy appears to be unsatisfactory for neuropathic ulcers. Several domestic and foreign studies were conducted looking for alternative to treat chronic plantar ulcers in leprosy (CPUL). Herbal plants that are easily affordable are expected to be an alternative wound care for MH's plantar pedis ulcers. Tea tree (Melaleuca alternifolia) is a native plant of the northeast coast of New South Wales in Australia. Tea tree comes from the Myrtaceae family, with yellow or purplish flowers and needle-like leaves, shaped like a bush which can also be used as an alternative for wound healing. ${ }^{2,3}$ Tea Tree Oil (TTO) is extracted from steam distillation of the leaves and twigs of the Australian native shrub Melaleuca alternifolia. TTO which is used for therapy is clear or pale yellow in color with a fresh odor and does not cause irritation to the skin. TTO products have been studied in recent years for their use as antimicrobial, antiviral, and anti-fungal as well as their effect on stimulating the immune system and enhancing immunity. ${ }^{4}$ As a whole, the components attached to TTO consist of $\alpha$-terpinene, $\alpha$ terpinolene, and $\alpha$-terpinen. $\alpha$-terpinene has the best levels of antioxidants when compared to a-terpinolene and c-terpinene. ${ }^{5}$ 
The TTO preparation in the form of hydrogel is believed to help the wound healing process. In a study comparing the effectiveness of TTO hydrogel (Levtrade International (Pty) Ltd.) with running water in 10 pigs with burns, it was shown that the wound healing results were faster in the TTO hydrogel group which was immediately applied from the time of injury to 21 days of use compared to control. The cold effect on hydrogel is effective in reducing tissue damage and accelerating wound healing. ${ }^{6}$

\section{METHODS}

This was an experimental prospective cohort study conducted by observing the clinical improvement of CPUL after topical TTO hydrogel 5\% therapy. The sample of the study was 22 CPULs (both single and multiple ulcers). The criteria were adult patients with ulcers $>6$ weeks, minimum ulcer size of $9 \mathrm{~cm}^{2}$ and $\leq$ $0.5 \mathrm{~cm}$ depth, good general conditions, and willing to participate in the study by signing the informed consent. The exclusion criteria were systemic corticosteroid medication in the past two weeks, patients with hemophilia/blood clotting disorder/using anti-platelet, hypersensitivity to adhesive plaster, and diabetes mellitus.

Debridement was performed before treatment. Once the ulcer clean, the size and depth were measured, and it was treated with topical TTO hydrogel $5 \%$. The ulcers were covered with transparent film dressings and opened every three days for clinical examination and TTO hydrogel 5\% treatment. The subjects visited the Dermatology and Venereology Outpatient Clinic in Dr. Soetomo General Academic Hospital Surabaya every week for ulcer treatment with TTO hydrogel 5\% and examination. The subjects should attend the follow-up treatment and examination for two months maximum. Therefore, should the participant recover in less than two months and no side effect observed, the follow-up was no longer needed. This research has been reviewed and approved by the Ethics Committee at Dr. Soetomo General Academic Hospital.

\section{RESULT}

The distribution of gender characteristics in the subjects of this study was more female $(59.1 \%)$ than male $(40.9 \%)$. All subjects in this study were aged 21 years and over, where the 21-50 age group was the same as those aged $>50$ years, namely as much as $50 \%$, with the mean age in this study subject was $49 \pm 6$ years. Most of the research subjects $(68.2 \%)$ had jobs that did not require a lot of standing / walking for a long time, such as housewives, raising chickens, motorized rickshaw pullers and also did not do much work outside the home due to the pandemic.

Table 1. Demographic data of study patients with chronic plantar ulcer of leprosy which had TTO hydrogel 5\% applied at the Leprosy Division Dermato-venereology Outpatient Clinic, Dr. Soetomo General Academic Teaching Hospital Surabaya

\begin{tabular}{ll}
\hline Variables & $\mathrm{n}(\%)$ \\
\hline Gender & $9(40.9)$ \\
Male, $\mathrm{n}(\%)$ & $13(59.1)$ \\
Female, $\mathrm{n}(\%)$ & $11(50.0)$ \\
\hline Age $($ years $)$ & $11(50.0)$ \\
$21-50$ years, $\mathrm{n}(\%)$ & \\
$>50$ years, $\mathrm{n}(\%)$ & $7(31.8)$ \\
\hline Occupation & $15(68.2)$ \\
A lot of standing/walking for a long time, $\mathrm{n}(\%)$ & \\
Rarely standing/walking for a long time, $\mathrm{n}(\%)$ & $6(27.2)$ \\
\hline Period of ulcer (years) & $16(72.8)$ \\
$<1$ year, $\mathrm{n}(\%)$ & $0(0)$ \\
$1-5$ years, $\mathrm{n}(\%)$ & $1(4,5)$ \\
$>5$ years, $(\%)$ & $10(45,5)$ \\
\hline Size of initial ulcer & $11(50,0)$ \\
$1 \mathrm{~cm}{ }^{2}$ & \\
$1-4 \mathrm{~cm}^{2}$ & $6(27,3)$ \\
$5-9 \mathrm{~cm}^{2}$ & $5(22,7)$ \\
Depth of initial ulcer & $5(22,7)$ \\
0.2 & $6(27,3)$ \\
0.3 & \\
0.4 & \\
0.5 &
\end{tabular}

$\mathrm{cm}$ : centimeter

TTO: Tea Tree Oil 
The work of the research subjects required a lot of standing / walking for a long time (31.8\%), including working as scavengers, rickshaw pullers, farmers, and construction workers who still worked during the pandemic. Most of the plantar ulcers in the research subjects had been going on for a long time, namely 1-5 years in 16 people $(72.8 \%),<1$ year in 6 people $(27.2 \%)$, with the average length of the ulcers in the subjects of this study was $13.36 \pm 8.27$ months. Data on the basic characteristics of research subjects can be seen in table 1 .

The mean percentage of ulcer healing every week until the end of treatment both in area and in ulcers was calculated from the mean area and in the initial ulcer area minus the mean area and in the $8^{\text {th }}$ week ulcer, divided by the mean area and in the initial ulcer, then multiplied by $100 \%$. Table 2 shows the mean percentage of ulcer healing that has increased every week. At the end of the study (week 8), the mean percentage reduction in ulcer area was $89.96 \pm 21.76$, and the mean percentage reduction in ulcers was 84.77 \pm 30.26 .

The clinical progress of ulcers in study subjects at the end of treatment was classified as cured, improved, persisted, or worsened. In the study subjects, it was found that ulcers were healed in 13 people $(59 \%)$, ulcers that improved in 8 people $(36.4 \%)$, ulcers that remained in 1 person $(4.6 \%)$, and there were no ulcers that got worse. Clinical ulcer progress data are presented in Table 3.

Processing data in this study using the Shapiro Wilk test to determine the distribution of normal data or not. In this study, the data obtained were both broad and in the ulcer not normally distributed ( $p<0.05$ ), so the statistical test to be used was a non-parametric statistical test with the Wilcoxon test. Table 5 presents a comparative test of the difference in mean ulcer sizes before and after treatment. Statistical tests on research subjects found significant differences both for the difference in ulcer area $(p=0.000)$ and for the difference in ulcers $(p=0.000)$ before and after treatment.

Table 2. Recovery percentage of chronic plantar ulcer of leprosy size and depth after treatment with TTO hydrogel

The recovery percentage of chronic plantar ulcer in leprosy TTO hydrogel $(\mathrm{n}=22)$

\begin{tabular}{lc}
\hline The average percentage of ulcer size reduction $(\%)$ & $21,77 \pm 17,38$ \\
Week I \pm SD & $38,66 \pm 29,07$ \\
Week II \pm SD & $55,45 \pm 28,93$ \\
Week III \pm SD & $68,80 \pm 28,79$ \\
Week IV \pm SD & $77,48 \pm 27,04$ \\
Week V \pm SD & $83,18 \pm 25,75$ \\
Week VI \pm SD & $87,16 \pm 22,90$ \\
Week VII \pm SD & $89,96 \pm 21,76$ \\
Week VIII \pm SD & \\
\hline Average percentage of ulcer depth reduction $(\%)$ & $15,91 \pm 19,23$ \\
Week I \pm SD & $34,55 \pm 27,89$ \\
Week II \pm SD & $51,59 \pm 32,28$ \\
Week III \pm SD & $61,14 \pm 31,08$ \\
Week IV \pm SD & $71,74 \pm 32,61$ \\
Week V \pm SD & $80,53 \pm 31,39$ \\
Week VI \pm SD & $82,05 \pm 31,50$ \\
Week VII \pm SD & $84,77 \pm 30,26$ \\
Week VIII \pm SD &
\end{tabular}

TTO: Tea Tree Oil, SD: Standard Deviation

Table 3. Clinical improvement of the patients with chronic plantar ulcer of leprosy at the end of the research

\begin{tabular}{lc}
\hline Clinical improvement of ulcer at the end of the research & $\begin{array}{c}\text { TTO hydrogel } \\
(\mathrm{n}=22)\end{array}$ \\
\hline Recovered, $\mathrm{n}(\%)$ & $13(59)$ \\
Improved, $\mathrm{n}(\%)$ & $8(36,4)$ \\
Constant, $\mathrm{n}(\%)$ & $1(4,6)$ \\
Worsen, $\mathrm{n}(\%)$ & $0(0)$ \\
\hline
\end{tabular}


Table 4. The comparative test for the size of chronic plantar ulcer of leprosy before and after the treatment

\begin{tabular}{llll}
\hline \multicolumn{2}{l}{ Size of ulcer } & $\begin{array}{l}\text { TTO hydrogel }(\mathrm{n}=22) \\
\text { Means } \pm \mathrm{SD}\end{array}$ & $\mathrm{p}$ Values \\
\hline \multirow{2}{*}{ Size } & Before & $4,24 \pm 2,33$ & \\
& After & $2,27 \pm 3,33$ & $\mathrm{P}=0.000^{*}$ \\
\hline \multirow{2}{*}{ Depth } & Before & $0,34 \pm 0,12$ & \\
& After & $0,20 \pm 0,20$ & $\mathrm{P}=0.000^{*}$ \\
\hline
\end{tabular}

*Significant differences $(\mathrm{p}<0.05)$, SD: Standard deviation, TTO: Tea Tree Oil

\section{DISCUSSION}

This study aimed to determine the efficacy of TTO hydrogel in the healing of chronic plantar ulcers of Morbus Hansen's with 22 subjects studied before and after the procedure. Each study subject was debrided before starting treatment using TTO hydrogel with the aim that the ulcers returned to the same healing phase at the start of the study, through the coagulation and inflammation phases. Treatment is carried out for 8 weeks with the application of TTO hydrogel every 3 days to match the wound healing phase starting from day 4 after wound debridement has entered the proliferation phase. All subjects completed up to 8 weeks of treatment so there was no dropout.

The results of this study indicated that more female subjects suffered from CPUL (59.1\%) with a mean age of $49.3 \pm 5.96$ years. The age of this research subject is in accordance with the age of the research subject in various studies on ulcers in $\mathrm{MH}$ patients including a study by Desancha et al which examined the health quality of $\mathrm{MH}$ ulcers which had an average age of 45 years. $^{7}$ Age is thought to have an influence on wound healing. Swift et al. conducted a study on young and old mice, there was a delay in the reepithelialization process, collagen synthesis, and angiogenesis compared to young mice. The ability to heal wounds with age also changes and these changes occur in all phases of wound healing, namely increased platelet aggregation, increased secretion of inflammatory mediators, late infiltration of macrophages and lymphocytes, impaired macrophage function, decreased growth factor secretion, late reepithelialization, angiogenesis and delayed collagen deposition, decreased collagen turnover and remodeling, and decreased wound strength. ${ }^{8}$ As we get older, there is an increase in the incidence of mitochondrial DNA mutations which is marked by an increase in the number of dysfunctional mitochondria accompanied by a decrease in the ability to eliminate them so that there is an increase in Reactive Oxygen Species (ROS) levels which can delay the wound healing process. ${ }^{9}$ Anova test in this study on age obtained $p=0.386$, which is that there is no relationship between age and TTO hydrogel, this can be related to the antioxidant effect of TTO which can reduce ROS by inhibiting ROS formation enzymes (xanthine oxidase, cyclooxygenase, and lypooxygenase) and influencing Nitric oxide production through the interaction of nitric oxide synthase (NOS), so that it can accelerate the process of wound healing.

As many as $68.2 \%$ of the subjects in this study did not have much activity or did heavy work during the treatment, while the remaining $31.8 \%$ of the subjects still did heavy activities or work. Occupation is one of the important risk factors in the healing process of plantar ulcers because immobilization is one way of managing plantar ulcers so that patients who are still doing heavy work or activities will take longer to heal their wounds than patients who immobilize or do not do much activity. Most of the subjects in this study did not do a lot of activities or heavy work because in addition to the research subjects, there were more women who worked as housewives who were also added to the current Covid-19 pandemic so that many male subjects did not do much activity. There is a relationship between the incidence of ulcers and the amount of pressure in the area of the foot where the ulcer is present, the higher the pressure on a certain area of the foot, the more risk of ulcers. The amount of pressure on the feet is affected by body weight, foot surface, motion of the joints and big toe, amputation, degree of anesthesia, severity of neuropathy, deformity and hypomobility. The longer a person stands / walks and the greater the body weight, the greater the pressure on the feet. In MH patients with neuropathic disorders do not change the position or rest the area, so that the large pressure continues. In $\mathrm{MH}$ patients there is also an autonomic disorder that causes impaired venivasomotor reflex which controls the increase in venous pressure when standing by increasing precapillary resistance so that blood flow can be normal. The loss of this reflex results in an increase in venous pressure resulting in tissue edema which can prevent wound healing. ${ }^{1}$ In this study, the Chi square test on the type of work found $p=1,000$ on ulcer healing, which means that there was no statistically significant relationship between the type of activity and 
ulcer healing. Subjects with plantar ulcers who have long standing / strenuous activities are thought to experience longer wound healing than subjects with light activity. Reducing or removing the load on the feet (off-loading) is an important factor in the successful management of chronic plantar ulcers. Offloading can include bed rest, use of crutches, wheelchairs, walkers or special footwear. The limitation of our study is that there is no off-loading on the research subjects. Subjects are only advised to reduce standing or walking activities. This aims to minimize the bias caused by excessive use of the feet to the time required for wound healing which could affect the results of the study. ${ }^{1}$

In this study, clinically the results of ulcers healed were $59 \%$, ulcers improved by $36.4 \%$, persistent ulcers were $4.6 \%$, and there were no ulcers that got worse. The mean percentage of ulcer healing at the end of the study was $89.96 \% \pm 21.76 \%$ for ulcer area and $81.74 \%$ $\pm 30.25 \%$ for deep ulcers. Research on the efficacy of herbal plants or essential oils in chronic $\mathrm{MH}$ plantar pedis ulcers has been conducted using Epigallocatechin gallate (EGCG) 1\% topical ointment. A total of 22 study subjects with plantar pedic ulcers were given EGCG 1\% topical ointment for 8 weeks and obtained significant clinical and statistical improvements.

TTO preparations in the form of hydrogel are said to be effective in helping the process of accelerating wound healing time in both acute and chronic wounds. Hydrogel has characteristics suitable for topical use. ${ }^{5}$ In a study comparing the effectiveness of TTO hydrogel (Levtrade International (Pty) Ltd.) with running water in 10 pigs with burns, it was shown that the wound healing results were faster in the TTO hydrogel group which was immediately applied from the time of injury to 21 days of use compared to control. The cold effect on hydrogel is effective in reducing tissue damage and accelerating wound healing. ${ }^{6}$

In this study, there were significant results both in the area of the wound $(\mathrm{p}=0.000)$ and in the wound $(\mathrm{p}$ $=0.000$ ) after topical TTO hydrogel therapy. This can be attributed to the anti-inflammatory role of TTO which has been proven in vitro and in vivo to influence the immune response. One of the water-soluble components of TTO can inhibit lipopolysaccharide which induces the production of pro-inflammatory mediators and terpinen-4-ol components which have been shown to affect the production of Tumor Necrotizing Factor (TNF)- $\alpha$, IL-1, IL-8, IL-10 and prostaglandin E2 with lipopolysaccharide. -activated monocytes. In chronic wounds there is also a continuous inflammatory process that results in ROS. An in-vitro study using 2 kinds of tests on the antioxidant function of TTO states that when compared to other natural antioxidants, TTO inhibits free radicals and inhibits fat peroxidase. This may be due to the inherent activity of several components present in TTO which can inhibit or reduce the aerobic oxidation ratio. ${ }^{11}$

TTO can have an effect on wound healing because of its antibacterial and antiseptic activities. According to Cox et al. (2000), the antimicrobial activity of the $M$. alternifolia terpenes is associated with their strong hydrophobicity. Here, the hydrophobic terpenes interact strongly with the membrane lipids of the pathogenic microorganisms, which affect the permeability of the membrane. ${ }^{12}$ Terpinen-4-ol, linalool, and alphaterpineol were the substances most widely mentioned to have antimicrobial activity in TTO. Terpinen-4-ol has been shown in clinical trials to act on cell walls. Infection control can also accelerate wound healing. ${ }^{13}$ The compound terpinene-4-ol, also identified amongst the main constituents of the Iranian Cymbopogon Olivieri essential oil, has been implicated in the antimicrobial activity against Gram-positive bacteria, Gram-negative bacteria, and the yeast Candida albicans. ${ }^{14}$

The literature states that the appropriate TTO level for wound care ranges from $1-10 \%$. Lee and colleagues conducted a study on 16 geriatric patients who had open wounds with Methicillin Resistant Staphylococcus aureus (MRSA) bacterial colonization using TTO $10 \%$ and there were no side effects that occurred in all of the study subjects. ${ }^{15}$ It has been reported that the incidence of allergic contact dermatitis to TTO is $5-10 \%$ when using TTO with levels greater than $10 \%$.

In this study, using TTO with a level of 5\% because this research is the first research conducted to minimize the possibility of side effects. It is proven that in this study the 5\% TTO used did not cause side effects in all study subjects and effective in the healing process of CPUL. The limitation of this study is that it does not do immobilization specifically including the use of assistive devices such as special shoes designed according to the shape of the feet of the research subject.

\section{REFERENCES}

1. Agale SA. Chronic Leg Ulcers: Epidemiology, Aetiopathogenesis,and Management. Ulcers 2013; 2013:1-9.

2. Ali, B., Al-Wabel, N. A., Shams, S., Ahamad, A., Khan, S. A., \& Anwar, F. Essential oils used in aromatherapy: A systemic review. Asian Pac J 
Trop Biomed 2015; 5(8):601-11. https://doi.org/10.1016/j.apjtb.2015.05.007

3. S. Siddique, Z. Perveen, S. Nawaz, K. Shahzad, and Z. Ali, "Chemical Composition and Antimicrobial Activities of Essential Oils of Six Species from Family Myrtaceae," J Essent OilBear Plants 2015. 18:950-56.

4. Pazyar, N., Yaghoobi, R., Bagherani, N., \& Kazerouni, A. Review: A review of applications of tea tree oil in dermatology. Int $\mathrm{J}$ Dermatol 2015; 52:784-90.

5. Kim HJ, Chen F, Wu C, Wang Xi, Chung HY, Jin Z. Evaluation of antioxidant activity of Australian tea tree (Melaleuca alternifolia) oil and its components. J Agric Food Chem 2014; 52:284954.

6. Jandera V, Hudson DA, de Wet PM, Innes PM, Rode H. Cooling the burn wound: evaluation of different modalities. Burns 2010; 26:265-70.

7. Desancha M, Kiranjha, W. A. Health beliefs surrounding leprosy induced foot ulceration; an exploratory qualitative study from South Nepal. Lepr Rev 2015; 86:254-64.

8. Guo S, D. L. Factors affecting wound healing. J Dent Res 2010; 89(3):219-29.

9. Tamama K, K. S. Acceleration of Wound Healing by Multiple Growth Factors and Cytokines Secreted from Multipotential Stromal
Cells/Mesenchymal Stem Cells. Adv Wound Care 2012; 4:177-82.

10. C. R. S. Prakoeswa, R. N. Oktaviyanti, D. M. Indramaya, E. Hendradri, S., \& Sawitri, L. Astari, D. D. \& M. Y. L. Efficacy of topical epigallocatechin gallate (EGCG) $1 \%$ on the healing of chronic plantar ulcers in leprosy. $\mathrm{J}$ Dermatolog Treat 2020.

11. Zhang, X., Guo, Y., Guo, L., Jiang, H., \& Ji, Q. In Vitro Evaluation of Antioxidant and Antimicrobial Activities of Melaleuca alternifolia Essential Oil, Biomed Res Int 2018; 2018.

12. S. D. Cox, C. M. Mann, J. L. Markham et al., "The mode of antimicrobial action of the essential oil of Melaleuca alternifolia (tea tree oil)," J Appl Microbiol 2012; 88:170-75.

13. Makvandi, S., Mo, A., \& Aminfar, S. The effect of local gentamicin solution on episiotomy healing: A randomized controlled clinical trial. Iran. J. Obstet 2014; 16:21-8.

14. M. Burits and F. Bucar, "Antioxidant activity of Nigella sativa essential oil," Phytother Res 2012; 14:323-38.

15. Lee, R. L. P., Leung, P. H. M., \& Wong, T. K. S. A randomized controlled trial of topical tea tree preparation for MRSA colonized wounds. Int. J. Nurs. Sci. 2014; 1(1):7-14. https://doi.org/10.1016/j.ijnss.2014.01.001 